Communications in Physics, Vol. 22, No. 4 (2012), pp. 327-336

\title{
ELECTRON DISTRIBUTION IN AlGaN/GaN MODULATION-DOPED HETEROSTRUCTURES
}

\author{
DINH NHU THAO \\ Center for Theoretical and Computational Physics, \\ College of Education, Hue University \\ NGUYEN THANH TIEN \\ College of Science, Can Tho University
}

\begin{abstract}
We present a calculation of the distribution of two-dimensional electron gas (2DEG) along the quantization direction in an AlGaN/GaN modulation-doped heterostructure (MDHS). The main confinement sources from ionized donors, 2DEG and polarization charges are properly taken into account. We show that the 2DEG distribution near the MDHS interface depends strongly on the model of potential barrier in use. Within the ideal model of infinite potential barrier, the 2DEG distribution near the interface is increased with a rise of the sheet densities of 2DEG and polarization charges. On the contrary, this distribution is decreased within the realistic model of finite potential barrier. Since the key mechanisms limiting the 2DEG mobility in MDHS are alloy disorder and surface roughness scatterings that are very sensitive to the near-interface 2DEG distribution, with a rise of the sheet densities of 2DEG and polarization charges the 2DEG mobility is decreased within the infinite-barrier model, while increased within the finite one.
\end{abstract}

\section{INTRODUCTION}

Group-III nitride-based heterostructures (HSs) have attracted many intense investigations because of their promising potential for high-voltage, high-power, and hightemperature microwave applications. [1] The mobility of two-dimensional electron gas (2DEG) is a characteristic property of the performance of high electron mobility transistor structures, [2] and it, in AlGaN/GaN HSs, depends strongly on their parameters such as temperature, 2DEG density, and alloy composition.

As well known, [1] polarization is an important property of nitride-based HSs. The polar HSs possess a high sheet density of polarization charges bound on the interface. Recently, we have shown [3] that the interface polarization charges take the three-fold role as the ionized impurities do. These charges on a rough interface are a carrier supply source into HSs, but also a confining source as well as a scattering mechanism for the carriers in polar HSs. The 2DEG mobility in AlGaN/GaN polar MDHSs was measured and calculated in decades. [4-12] However, there are several drawbacks in the previous calculations. $[4,7,8,10,11]$ The interface polarization charges were taken into account only as a carrier supply source, but often ignored as confining and scattering sources. In addition, the ionized impurities as a confining source were also omitted.

The aim of this paper is to present a theoretical study on electron distribution in $\mathrm{AlGaN} / \mathrm{GaN}$ MDHSs that takes properly the effects of all possible confining sources into 
account. The paper is organized as follows. In Sec. II, the electron distribution in Al$\mathrm{GaN} / \mathrm{GaN}$ MDHSs is determined by all confining sources, inclusive of interface polarization charges. In Sect. III, we formulate the basic equations for calculation of low-temperature transport in MDHSs limited by AD and SR scattering. In Sect. IV, we examine the dependence of 2DEG mobility on the sheet polarization charge density as well as 2DEG density observed in AlGaN/GaN and AlN/GaN MDHSs. At last, a summary is given in Sec. V.

\section{ELECTRON DISTRIBUTION IN MDHSs}

\section{II.1. Variational wave function in HSs of finite potential barrier}

At low temperature, the 2DEG is assumed to primarily occupy the lowest subband. It was shown [13-15] that for a finitely deep triangular quantum well, the 2DEG distribution may be very well described by a Fang-Howard wave function, [16]

$$
\zeta(z)= \begin{cases}A \kappa^{1 / 2} \exp (\kappa z / 2) & \text { for } z<0 \\ B k^{1 / 2}(k z+c) \exp (-k z / 2) & \text { for } z>0 .\end{cases}
$$

Here $\kappa$ and $k$ are half the wave numbers in the barrier and channel layers, respectively. The envelope wave function in Eq. (1) exhibits a peak $\zeta_{\text {peak }}=\zeta\left(z_{\text {peak }}\right)$ at

$$
z_{\text {peak }}=\frac{2-c}{k} \text {. }
$$

The wave function of the lowest subband, namely its wave vectors $k$ and $\kappa$, is to minimize the total energy per electron, which is determined by the Hamiltonian:

$$
H=T+V_{\text {tot }}(z)
$$

where $T$ is the kinetic energy, and $V_{\text {tot }}(z)$ is the overall confining potential.

\section{II.2. Confining potentials in polar MDHSs}

The carrier confinement in a polar HS is fixed by all possible confining sources located along the growth direction, viz., potential barrier, interface polarization charges, Hartree potential created by ionized impurities and 2DEG:

$$
V_{\mathrm{tot}}(z)=V_{\mathrm{b}}(z)+V_{\sigma}(z)+V_{\mathrm{H}}(z) .
$$

The potential barrier of some finite height $V_{0}$ located at $z=0$ reads as [3]

$$
V_{\mathrm{b}}(z)=V_{0} \theta(-z)
$$

with $\theta(z)$ as a unity step function. It is well known $[1,17-20]$ that the potential due to positive polarization charges bound on the interface given by [3]

$$
V_{\sigma}(z)=\frac{2 \pi}{\varepsilon_{\mathrm{a}}} e \sigma|z|
$$

with $\sigma$ as their total sheet density. Here $\varepsilon_{\mathrm{a}}=\left(\varepsilon_{\mathrm{b}}+\varepsilon_{\mathrm{c}}\right) / 2$ is the average value of the dielectric constants of the barrier $\left(\varepsilon_{\mathrm{b}}\right)$ and the channel $\left(\varepsilon_{\mathrm{c}}\right)$. Next, we calculate the Hartree potential induced by the ionized donors and 2DEG in the HS. The Hartree potential may be represented in the form $[5,9,12,14,15,21,23]$

$$
V_{\mathrm{H}}=V_{\mathrm{I}}+V_{\mathrm{s}} \text {. }
$$


The first term is the potential due to remote donors

$$
V_{\mathrm{I}}(z)=E_{\mathrm{I}}+\frac{4 \pi e^{2} n_{\mathrm{I}}}{\varepsilon_{\mathrm{a}}} \begin{cases}0 & \text { for } z<-z_{\mathrm{d}}, \\ \left(z+z_{\mathrm{d}}\right)^{2} / 2 L_{\mathrm{d}} \text { for }-z_{\mathrm{d}}<z<-z_{\mathrm{s}}, \\ z+\left(z_{\mathrm{d}}+z_{\mathrm{s}}\right) / 2 \text { elsewhere. }\end{cases}
$$

Here the sheet donor density is $n_{\mathrm{I}}=N_{\mathrm{I}} L_{\mathrm{d}}$ with $N_{\mathrm{I}}$ is the bulk (per unit volume) density of donors. The $z_{\mathrm{s}}=L_{\mathrm{s}}$ and $z_{\mathrm{d}}=L_{\mathrm{s}}+L_{\mathrm{d}}$, with $L_{\mathrm{s}}$ and $L_{\mathrm{d}}$ as the thicknesses of the spacer and doping layers, respectively.

The second term is the potential due to 2DEG, determined by the sheet electron density $n_{\mathrm{s}}$ and their distribution, i.e., the variational parameters entering the electron wave function as follows:

$$
V_{\mathrm{s}}(z)=-\frac{4 \pi e^{2} n_{\mathrm{s}}}{\varepsilon_{\mathrm{a}}} \begin{cases}f(z) & \text { for } z<0 \\ g(z)+z+f(0)-g(0) & \text { for } z>0\end{cases}
$$

where by definition:

$$
f(z)=\frac{A^{2}}{\kappa} e^{\kappa z}
$$

and

$$
g(z)=\frac{B^{2}}{k} e^{-k z}\left[k^{2} z^{2}+2 k(c+2) z+c^{2}+4 c+6\right] .
$$

\section{II.3. Total energy per electron in the lowest subband}

We now turn to the total energy per electron for the 2DEG occupying the groundstate subband. The expectation value of the Hamiltonian reads as [33]

$$
E_{0}(k, \kappa)=\langle T\rangle+\left\langle V_{\mathrm{b}}\right\rangle+\left\langle V_{\sigma}\right\rangle+\left\langle V_{\mathrm{I}}\right\rangle+\left\langle V_{\mathrm{s}}\right\rangle .
$$

For the kinetic energy, with the use of the wave function from Eq. (1), it holds:

$$
\langle T\rangle=-\frac{\hbar^{2}}{8 m_{z}}\left[A^{2} \kappa^{2}+B^{2} k^{2}\left(c^{2}-2 c-2\right)\right],
$$

where $m_{z}$ is the out of-plane effective mass of the GaN electron. And for the potentials related to the barrier and the polarization charges bound on the interface, we have

$$
\left\langle V_{\mathrm{b}}\right\rangle=V_{0} A^{2},
$$

and

$$
\left\langle V_{\sigma}\right\rangle=\frac{2 \pi e \sigma}{\varepsilon_{\mathrm{a}}}\left[\frac{A^{2}}{\kappa}+\frac{B^{2}}{k}\left(c^{2}+4 c+6\right)\right] .
$$

Next, the average potential due to charged impurities is given by

$$
\begin{array}{r}
\left\langle V_{\mathrm{I}}\right\rangle=E_{\mathrm{I}}+\frac{4 \pi e^{2} n_{\mathrm{I}}}{\varepsilon_{\mathrm{a}}}\left\{\frac{d+s}{2 \kappa}+\frac{A^{2}}{\kappa(d-s)}\left[\chi_{2}(d)-\chi_{2}(s)\right.\right. \\
\left.-d \chi_{1}(d)+s \chi_{1}(s)+\frac{d^{2}}{2}\left[\chi_{0}(d)-1\right]-\frac{s^{2}}{2}\left[\chi_{0}(s)-1\right]\right] \\
\left.+\frac{B^{2}}{k}\left(c^{2}+4 c+6\right)\right\},
\end{array}
$$


with $s=\kappa L_{\mathrm{s}}$ and $d=\kappa\left(L_{\mathrm{d}}+L_{\mathrm{s}}\right)$ as the dimensionless doping sizes. Here we introduced an auxiliary function:

$$
\chi_{n}(x)=1-e^{-x} \sum_{l=0}^{n} \frac{x^{l}}{l !},
$$

with $n=0,1,2, \ldots$ as an integer. Lastly, for the 2DEG potential, it holds:

$$
\begin{aligned}
\left\langle V_{\mathrm{s}}\right\rangle= & -\frac{4 \pi e^{2} n_{\mathrm{s}}}{\varepsilon_{\mathrm{a}}}\left[\frac{A^{2}}{\kappa}-\frac{A^{4}}{2 \kappa}+\frac{B^{2}}{k}\left(c^{2}+4 c+6\right)\right. \\
& \left.-\frac{B^{4}}{4 k}\left(2 c^{4}+12 c^{3}+34 c^{2}+50 c+33\right)\right],
\end{aligned}
$$

For infinite confinement, the minimization of the total energy per electron leads to a simple expression for the channel wave vector in the ground state:

$$
k=\left\{\frac{24 \pi m_{z} e^{2}}{\hbar^{2} \varepsilon_{\mathrm{a}}}\left[(\sigma / e)+2 n_{\mathrm{I}}-\frac{15}{24} n_{\mathrm{s}}\right]\right\} .
$$

\section{LOW-TEMPERATURE ELECTRON MOBILITY IN AlGaN/GaN MDHS}

\section{III.1. Basic equations}

The electrons moving along the in-plane are scattered by various disorder sources, which are normally characterized by some random fields. Scattering by a Gaussian random field is specified by its autocorrelation function (ACF) in wave vector space $\left\langle|U(\mathbf{q})|^{2}\right\rangle$. [2] Hereafter, $U(\mathbf{q})$ is a $2 \mathrm{D}$ Fourier transform of the unscreened potential weighted with the lowest-subband wave function:

$$
U(\mathbf{q})=\int_{-\infty}^{+\infty} d z|\zeta(z)|^{2} U(\mathbf{q}, z)
$$

The inverse transport lifetime (scattering rate) at low temperatures are then represented in terms of the autocorrelation function for each disorder as follows: [24, 25]

$$
\frac{1}{\tau}=\frac{1}{2 \pi \hbar E_{\mathrm{F}}} \int_{0}^{2 k_{\mathrm{F}}} d q \frac{q^{2}}{\left(4 k_{\mathrm{F}}^{2}-q^{2}\right)^{1 / 2}} \frac{\left\langle|U(\mathbf{q})|^{2}\right\rangle}{\varepsilon^{2}(q)},
$$

Here, $\mathbf{q}$ denotes the momentum transfer by a scattering event in the interface plane, $q=|\mathbf{q}|=2 k_{\mathrm{F}} \sin (\theta / 2)$ with $\theta$ as an angle of scattering. The Fermi wave number is fixed by the sheet electron density: $k_{\mathrm{F}}=\sqrt{2 \pi n_{\mathrm{s}}}$, and $E_{\mathrm{F}}=\hbar^{2} k_{\mathrm{F}}^{2} / 2 m^{*}$, with $m^{*}$ as the in-plane effective mass of the GaN electron. The dielectric function $\varepsilon(q)$ is evaluated within the random phase approximation. $[2,15]$

At low temperatures $[10,11,23]$ the electrons in a polar MDHS are expected to experience the following main scattering mechanisms: (i) alloy disorder (AD) and (ii) surface roughness (SR). The overall transport lifetime is then determined by the ones for individual disorders in accordance with Matthiessen's rule:

$$
\frac{1}{\tau_{\text {tot }}}=\frac{1}{\tau_{\mathrm{AD}}}+\frac{1}{\tau_{\mathrm{SR}}} \text {. }
$$




\section{III.2. Alloy disorder (AD)}

The ACF for AD scattering is given in terms of the barrier wave number $\kappa$ as follows: $[3,13,14]$

$$
\left\langle\left|U_{\mathrm{AD}}(\mathbf{q})\right|^{2}\right\rangle=x(1-x) u_{\mathrm{al}}^{2} \Omega_{0} \frac{A^{4} \kappa}{2}\left[e^{-2 \kappa L_{\mathrm{a}}}-e^{-2 \kappa L_{\mathrm{b}}}\right] .
$$

Here $x$ is the alloy composition in the barrier, $L_{\mathrm{b}}$ is its thickness, $u_{\mathrm{al}}$ is the alloy potential $u_{\mathrm{al}} \sim \Delta E_{\mathrm{c}}(1),[14]$ and $\Omega_{0}$ is the volume occupied by one atom. [31]

It is to be noticed that for large enough barrier thicknesses, the second term in Eq. (23) is negligibly small. Thus, AD scattering is determined mainly by the first term proportional to $\zeta^{4}\left(z=-L_{\mathrm{a}}\right)$, i.e., by the value of the wave function near the interface.

\section{III.3. Surface roughness (SR)}

The ACF for surface roughness scattering is given as follows [2]

$$
\left\langle\left|U_{\mathrm{SR}}(\mathbf{q})\right|^{2}\right\rangle=\left|F_{\mathrm{SR}}(t)\right|^{2}\left\langle\left|\Delta_{\mathbf{q}}\right|^{2}\right\rangle \text {. }
$$

Here $F_{\mathrm{SR}}$ is the form factor for SR scattering connected with roughness of the potential barrier, determined as follows $[2,23,32]$

$$
F_{\mathrm{SR}}=\left\langle V_{\sigma}^{\prime}\right\rangle+\left\langle V_{\mathrm{I}}^{\prime}\right\rangle+\left\langle V_{\mathrm{s}}^{\prime}\right\rangle
$$

with $V^{\prime}=\partial V(z) / \partial z$. The calculation of the average forces is straightforward with the use of the lowest-subband wave function from Eq. (1). These read as follows.

For polarization charges of sheet density $\sigma$ :

$$
\left\langle V_{\sigma}^{\prime}\right\rangle=\frac{4 \pi e^{2}}{\varepsilon_{\mathrm{a}}} \frac{\sigma}{2 e}\left(1-2 A^{2}\right) .
$$

For remote ionized impurities of sheet density $n_{\mathrm{I}}$ :

$$
\begin{aligned}
\left\langle V_{\mathrm{I}}^{\prime}\right\rangle= & \frac{4 \pi e^{2} n_{\mathrm{I}}}{\varepsilon_{\mathrm{a}}}\left\{1-A^{2}-\frac{A^{2}}{d-s}\left[\chi_{1}(d)\right.\right. \\
& \left.\left.-\chi_{1}(s)-d \chi_{0}(d)+s \chi_{0}(s)\right]\right\} .
\end{aligned}
$$

For the 2DEG distribution of sheet density $n_{\mathrm{s}}$ :

$$
\begin{aligned}
\left\langle V_{\mathrm{s}}^{\prime}\right\rangle= & -\frac{4 \pi e^{2} n_{\mathrm{s}}}{\varepsilon_{\mathrm{a}}}\left[1-A^{2}+\frac{A^{4}}{2}-\frac{B^{4}}{2}\right. \\
& \left.\times\left(c^{4}+4 c^{3}+8 c^{2}+8 c+4\right)\right] .
\end{aligned}
$$

\section{RESULTS AND CONCLUSIONS}

In this section, we are dealing with the estimation of the confinement effect on the electron wave function from all electrostatic sources, viz., interface polarization charges, 2DEG, and remote ionized donors. It is well-known that a change in the alloy composition $x$ implies a change in many other quantities, such as the barrier height $V_{0}(x)$, sheet polarization charge density $\sigma(x), 2 \mathrm{DEG}$ density $n_{\mathrm{s}}(x)$, and, may be, roughness profile $\Delta(x)$, 
$\Lambda(x)$. $[9,12]$ Thus, for an apparent illustration in academic study, we assume provisionally one parameter varied, while the others fixed.

In the literature $[7,10-12,22,23]$ one often adopted the ideal model of infinite barrier, based on the standard Fang-Howard wave function. $[2,16]$ This simplified essentially mathematics of the transport theory and was a good approximation for some scattering mechanisms by, e.g., ionized impurities and phonons that are insensitive to the near-interface 2DEG distribution. However, in the case under consideration, the key mechanisms are AD and SR that are very sensitive thereto. Thus, we examine the confinement effect within the realistic model of finite barrier, based on the modified Fang-Howard wave function. [13] The barrier height in $\mathrm{AlGaN} / \mathrm{GaN}$ MDHS is provisionally fixed, assumed to be equal to the conduction band offset for $x=0.3: V_{0}=0.45 \mathrm{eV}$.

In Figs. 1 and 2, we display the modified Fang-Howard wave function $\zeta(z)$ under a modulation doping of bulk donor density $N_{\mathrm{I}}=6 \times 10^{18} \mathrm{~cm}^{-3}$, thickness for doping $L_{\mathrm{d}}=150 \AA$, and spacer $L_{\mathrm{s}}=70 \AA$. In Fig. 1, this is plotted for a 2DEG density $n_{\mathrm{s}}=0.5 \times 10^{13} \mathrm{~cm}^{-2}$ and various sheet polarization charge densities $\sigma / e=0,0.5,1,5$ $\left(10^{13} \mathrm{~cm}^{-2}\right)$, while in Fig. 2 for a polarization charge density $\sigma / e=10^{13} \mathrm{~cm}^{-2}$ and various 2DEG densities $n_{\mathrm{s}}=0,0.1,0.5,1\left(10^{13} \mathrm{~cm}^{-2}\right)$. In Fig. 3, we draw the modified FangHoward wave function for thickness for doping $L_{\mathrm{d}}=150 \AA$, spacer $L_{\mathrm{s}}=70 \AA$, a $2 \mathrm{DEG}$ density $n_{\mathrm{S}}=0.5 \times 10^{13} \mathrm{~cm}^{-2}$, a polarization charge density $\sigma / e=10^{13} \mathrm{~cm}^{-2}$ and various donor densities $N_{\mathrm{I}}=1,5,10 \times 10^{18} \mathrm{~cm}^{-3}$.

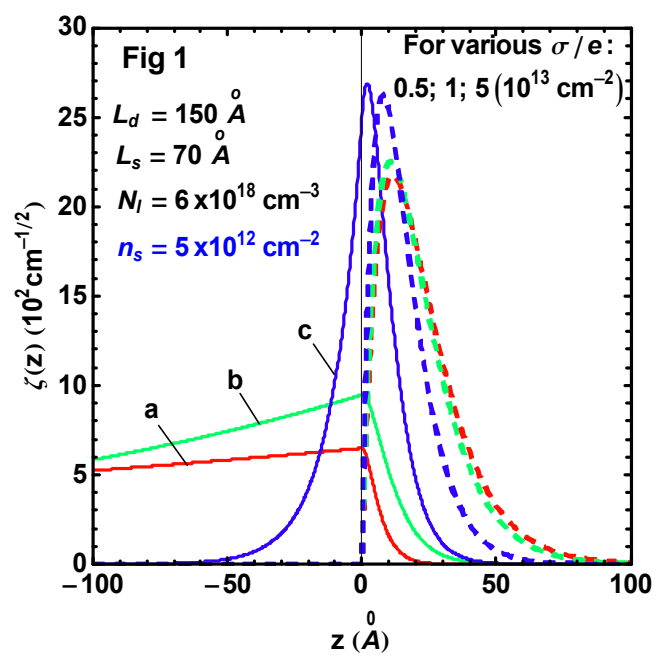

Fig. 1. Wave function $\zeta(z)$ in AlGaN/GaN MDHS for various sheet polarization charge densities $\sigma / e=5 \times 10^{12}, 10^{13}, 5 \times 10^{13} \mathrm{~cm}^{-2}$, labeled a, b, c, respectively. Solid lines refer to the finite-barrier model and dashed lines to the infinite one.

As seen from Figs. 1, 2, and 3 the peak of the electron wave function (2DEG peak), $\zeta_{\text {peak }}$, is located in a channel region near the interface plane. Figure 1 reveals that within the finite-barrier model the 2DEG peak is lifted with a rise of the sheet polarization charge density $\sigma$, but from Fig. 2, lowered with a rise of the 2DEG density $n_{\mathrm{s}}$. According to Eqs. 


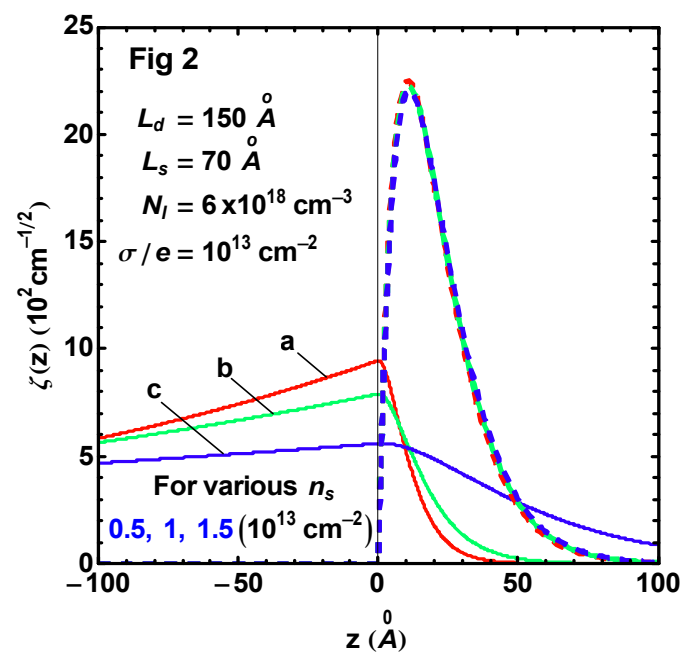

Fig. 2. Wave function $\zeta(z)$ in AlGaN/GaN MDHS for various sheet 2DEG densities $n_{\mathrm{s}}=10^{12}, 5 \times 10^{12}, 10^{13} \mathrm{~cm}^{-2}$, labeled a, b, c, respectively. The interpretation is the same as in Fig. 1.

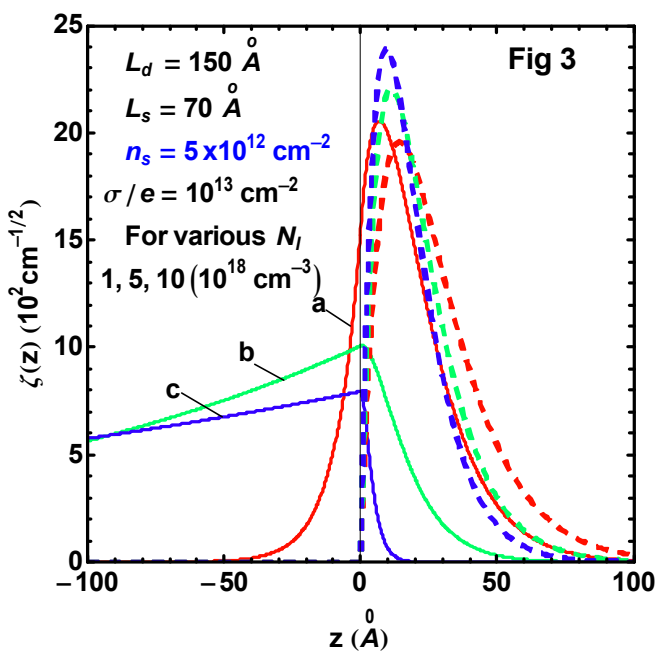

Fig. 3. Wave function $\zeta(z)$ in $\mathrm{AlGaN} / \mathrm{GaN}$ MDHS for various donor densities $N_{\mathrm{I}}=10^{18}, 5 \times 10^{18}, 10^{19} \mathrm{~cm}^{-3}$, labeled a, b, c, respectively. The interpretation is the same as in Fig. 1.

(1) and (2) the 2DEG peak is given by $\zeta_{\text {peak }}=(\sqrt{2} / \exp 1) k$. With $k$ from Eq. (19), this exhibits clearly the same behavior as in the infinite-barrier model. Figure 3 reveals that the 2DEG peak is lowered with a rise of the donor density $N_{\mathrm{I}}$ within the finite-barrier model, but lifted within the infinite-barrier one. 
The variation of the 2DEG peak with a rise of the charge density of some confining source is a result of combination of the opposite effects due to the potential barrier and the electrostatic force from this source. The latter effect depends on the position of its charges in respect to the 2DEG. An evident example is the attraction from ionized donors located in a space (barrier) different from that of the 2DEG (channel). The wave function is then shifted left (towards the barrier), thus because of the normalization of the wave function the 2DEG peak is lowered. On the other hand, the potential barrier prevents this left shift, the 2DEG is squeezed, thus its peak is lifted. In the finite-barrier model where the wave function can penetrate through the interface plane, the barrier effect is less than the donor one, so the 2DEG peak is lowered. However, in the infinite-barrier model where the penetration is impossible, the former is larger than the latter, so the 2DEG peak is lifted. This behavior is opposite to the lowered 2DEG peak in the case of uniform doping, [33] where the donors are located in the channel, i.e., the same space with the 2DEG.

Furthermore, owing to the repulsion among electrons the $z$-confinement is more relaxed with a rise of the 2DEG density, thus the 2DEG peak is lowered with a rise of $n_{\mathrm{s}}$ in both the barrier models as stated above. This is in contrast to the behavior of the wave function in the infinite-barrier model given earlier [7] that the 2DEG peak is lifted with larger $n_{\mathrm{s}}$. The 2DEG peak lifting was inferred from fitting of the electron wave function to the 2DEG mobility under the assumption that the mobility must be limited extra by interface impurities of a high density $\sigma_{\mathrm{II}} \sim 2 \times 10^{13} \mathrm{~cm}^{-2}$, but that concept was indicated $[34,35]$ to be very suspect even at a much lower interface impurity density $\sigma_{\text {II }} \sim 10^{11} \mathrm{~cm}^{-2}$.

At last, for $\sigma>0$ the polarization charges located on the interface plane can cause the attraction of electrons on two sides, in the channel and in the barrier. Due to the attraction of the barrier electrons towards the channel, the left shift of 2DEG is more prevented with larger $\sigma$. Hence, in both barrier models the $z$-confinement is more enhanced with a rise of $\sigma$, so the 2DEG peak is lifted.

Based on detail consideration, we concluded that the infinite barrier model is only applicable for scatterings that are insensitive to the near-interface 2DEG distribution, e.g., ionized impurities and phonons. For scatterings sensitive thereto, as alloy disorder and surface roughness, the finite barrier model must be applied.

\section{SUMMARY}

We calculated the 2DEG distribution along the quantization direction in an AlGaN/GaN MDHS. In the calculation we took into account all the main confinement sources from ionized donors, 2DEG and polarization charges. We then considered the dependence of the distribution on the sheet polarization charge density, the 2DEG density as well as donor density. We saw that the 2DEG distribution near the MDHS interface depends strongly on the model of potential barrier in use. Moreover, we saw its contrary behaviour with a change of the sheet densities of $2 \mathrm{DEG}$ and polarization charges, depending on the height of potential barrier is infinite or finite. 


\section{ACKNOWLEDGMENT}

The authors would like to thank Prof. Doan Nhat Quang very much indeed for his valuable guiding discussions.

\section{REFERENCES}

[1] Polarization Effects in Semiconductors: From Ab Initio Theory to Device Applications, edited by C. Wood and D. Jena (Springer, New York, 2008).

[2] T. Ando, A. B. Fowler, and F. Stern, Rev. Mod. Phys. 54 (1982) 437.

[3] D. N. Quang, N. H. Tung, and N. T. Tien, J. Appl. Phys. 109 (2011) 10.

[4] L. Hsu and W.Walukiewicz, Phys. Rev. B56 (1997) 1520.

[5] S. Keller, G. Parish, P. T. Fini, S. Heikman, C.-H. Chen, N. Zhang, S. P. DenBaars, U. K. Mishra, and Y.-F. Wu, J. Appl. Phys. 86 (1999) 5850.

[6] S. Keller, S. Heikman, L. Shen, I. P. Smorchkova, S. P. DenBaars, and U. K. Mishra, Appl. Phys. Lett. 80 (2002) 4387.

[7] J. Antoszewski, M. Gracey, J. M. Dell, L. Faraone, T. A. Fisher, G. Parish, Y.-F. Wu, and U.K Mishra, J. Appl. Phys. 87 (2000) 3900.

[8] L. Hsu and W. Walukiewicz, J. Appl. Phys. 89 (2001) 1783.

[9] S. Arulkumaran, T. Egawa, H. Shikawa, T. Jimbo, J. Vac. Sci. Tecnol. B21 (2003) 888.

[10] D. Zanato, S. Gokden, N. Balkan, B. K. Ridley, and W. J. Schaff, Semicond. Sci. Technol. 19 (2004) 427.

[11] M. N. Gurusinghe, S. K. Davidson, and T. G. Anderson, Phys. Rev. B72 (2005) 045316.

[12] M. Miyoshi, T. Egawa, H. Shikawa, J. Vac. Sci. Tecnol. B23 (2005) 1527.

[13] T. Ando, J. Phys. Soc. Jpn. 51 (1982) 3893; 51 (1982) 3900.

[14] G. Bastard, Wave Mechanics Applied to Semiconductor Heterostructures, Les Editions de Physique, Paris, 1988.

[15] Y. Okuyama and N. Tokuda, Phys. Rev. B40 (1989) 9744.

[16] F. F. Fang and W. E. Howard, Phys. Rev. Lett. 16 (1966) 797 .

[17] A. Bykhovski, G. Gelmont, and M. Shur, J. Appl. Phys. 74 (1993) 6734

[18] F. Bernardini, V. Fiorentini, and D. Vanderbilt, Phys. Rev. B56 (1997) R10 024 .

[19] I. P. Smorchkova, C. R. Elsass, J. P. Ibbetson, R. Vetury, B. Heying, P. Fini, E. Haus, S. DenBaars, J. S. Spect, and U. K. Mishra, J. Appl. Phys. 86 (1999) 4520.

[20] O. Ambacher, B. Foutz, J. Smart, J. R. Shealy, N. G. Weimann, K. Chu, M. Murphy, A. J. Sierakowski, W. J. Shaff, L. F. Eastman, R. Dimitrov, A. Mitchell, and M. Stutzmann, J. Appl. Phys. 87 (2000) 334 .

[21] R. Enderlein and N. J. M. Horing, Fundamentals of Semiconductor Physics and Devices, World Scientific, Singapore, 1997.

[22] K. Hirakawa and H. Sakaki, Phys. Rev. B 33 (1986) 8291.

[23] D. N. Quang, V. N. Tuoc, N. H. Tung, N. V. Minh, and P. N. Phong, Phys. Rev. B72 (2005) 245303.

[24] A. Gold and W. Götze, Phys. Rev. B 33 (1986) 2495.

[25] A. Gold, Phys. Rev. B35 (1987) 723.

[26] M. Jonson, J. Phys. C9 (1976) 3055.

[27] A. Fischer, H. Kühne, and H. Richter, Phys. Rev. Lett. 73 (1994) 2712.

[28] N. Maeda, T. Nishida, N. Kobayashi, and M. Tomizawa, Appl. Phys. Lett. 73 (1998) 1856.

[29] T.-H. Yu and K. F. Brennan, J. Appl. Phys. 89 (2001) 3827.

[30] D. Jena, S. Heikman, J. S. Speck, A. Gossard, and U. Mishra, Phys. Rev. B67 (2003) 153306.

[31] D. Jena, Y. P. Smorchkova, C. R. Elsass, A. C. Gossard, and U. K. Mishra, in Proceedings of the 25th International Conference on Physics of Semiconductors, edited by N. Miura and T. Ando, p. 771, Osaka, 2000.

[32] Y. Matsumoto and Y. Uemura, Jpn. J. Appl. Phys., Suppl. 2 (1974) 367. 
[33] D. N. Quang, N. H. Tung, V. N. Tuoc, N. V. Minh, H. A. Huy, and D. T. Hien, Phys. Rev. B74 (2006) 205312.

[34] F. Schäffler, Semicond. Sci. Technol. 12 (1997) 1515.

[35] R. J. P. Lander, M. J. Kearney, A. I. Horrel, E. H. C. Parker, P. J. Fillips, and T. E. Whall, Semicond. Sci. Technol. 12 (1997) 1604.

Received 30 September 2011. 